18,13

\title{
Влияние построчного лазерного сканирования на свойства лазерно-индуцированного графена
}

\author{
() К.Г. Михеев, Р.Г. Зонов, А.В. Сюгаев, Д.Л. Булатов, Г.М. Михеев \\ Удмуртский федеральный исследовательский центр УрО РАН, \\ Ижевск, Россия \\ E-mail: k.mikheev@udman.ru
}

Поступила в Редакцию 17 января 2022 г.

В окончательной редакции 17 января 2022 г.

Принята к публикации 21 января 2022 г.

Технология карбонизации полиимидной (ПИ) пленки путем прямой лазерной обработки привлекает большое внимание благодаря универсальности и простоте получения углеродного материала, лазерноиндуцированного графена (ЛИГ), используемого при создании различных датчиков и функциональных устройств. В настоящей работе получены пленочные структуры ЛИГ путем построчного сканирования пучка непрерывного $\mathrm{CO}_{2}$-лазера по поверхности ПИ-пленки. Синтезированный пленочный углеродный материал исследован с помощью оптической и сканирующей электронной микроскопии (СЭМ), рентгенофотоэлектронной спектроскопии (РФЭС) и спектроскопии комбинационного рассеяния света (КРС). Показано, что спектры КРС отдельной строки ЛИГ и множества строк ЛИГ, накладывающихся друг на друга, существенно отличаются друг от друга. Установлено, что многократное лазерное сканирование ПИ-пленки приводит к заметному уменьшению количества дефектов ЛИГ, а также к значительному уменьшению удельного поверхностного сопротивления синтезируемого пленочного материала. Полученные результаты могут быть использованы при синтезе пленочных структур ЛИГ с улучшенными характеристиками.

Ключевые слова: лазерно-индуцированный графен, полиимидная пленка, построчное лазерное сканирование.

DOI: 10.21883/FTT.2022.05.52341.277

\section{1. Введение}

В последние годы активно развиваются новые технологии, связанные с носимыми устройствами, беспроводной связью, которые влияют как на индустрию наносистем, так и на повседневную жизнь людей. С целью повышения эффективности и удобства в этих областях все больше используются гибкие материалы и различные микроустройства [1-4]. Для их изготовления широко применяются углеродные материалы, такие как графен, оксид графена и углеродные нанотрубки [5-8]. Одним из наиболее эффективных методов получения таких углеродных материалов является карбонизация различных прекурсоров благодаря своему удобству и экономичности. Карбонизация может осуществляться за счет химического, теплового, а также лазерного воздействия [9-11]. Среди этих способов прямое лазерное воздействие имеет такие преимущества как экономичность, возможность получения произвольной геометрической формы получаемого материала и отсутствие необходимости в специальных средах [12,13].

В качестве прекурсоров для лазерного пиролиза могут служить синтетические и природные полимеры $[7,11,14,15]$. Были разработаны различные устройства путем лазерной обработки этих материалов. Однако для массового производства микроустройств необходимы стабильные коммерчески доступные углеродные материалы. С этой точки зрения полиимид (ПИ) является иде- альным прекурсором для лазерного пиролиза, одновременно выполняющим функцию материала подложки. ПИ является доступным материалом, отличается гибкостью и термостойкостью, устойчивостью к большинству растворителей и часто используется для электрической и тепловой изоляции [16]. Именно на ПИ-пленке в 2014 г. была впервые получена графеновая пленочная структура при построчном сканировании сфокусированного импульсно-периодического излучения $\mathrm{CO}_{2}$-лазера [17], которая получила название ,лазерно-индуцированный графен“ (ЛИГ). С этого момента было опубликовано огромное количество научных работ, связанных с этим материалом, ввиду его преимуществ при создании микросуперконденсаторов, фильтров, мембран, датчиков газа и др. [18-24]. В подавляющем большинстве работ ЛИГ синтезировался с применением импульсно-периодических лазеров, работающих в различных спектральных диапазонах [25-27]. Импульсно-периодическое лазерное воздействие при не очень высоких частотах (например, для $\mathrm{CO}_{2}$-лазеров около $6 \mathrm{kHz}$ [28]) на ПИ-пленку не может обеспечить однородность синтезируемой пленочной структуры ЛИГ на поверхности пленки. В связи с этим представляет интерес применение непрерывного излучения $\mathrm{CO}_{2}$-лазера для формирования более равномерной пленочной структуры ЛИГ. В наших недавних работах мы показали, что ЛИГ может быть синтезирован на поверхности ПИ-пленки с помощью непрерывного излучения $\mathrm{CO}_{2}$-лазера $[29,30]$. В частности, было по- 


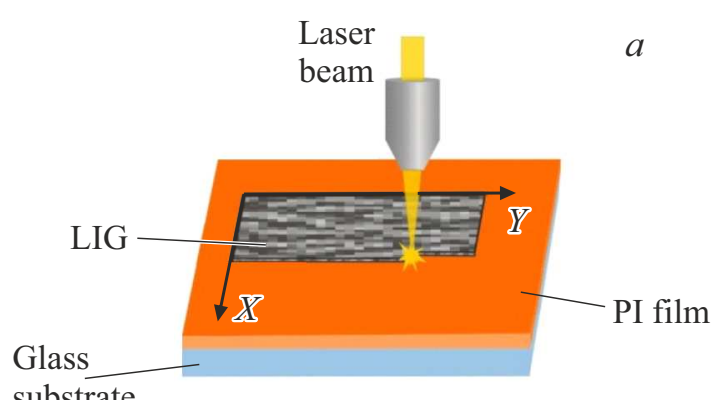

$c$

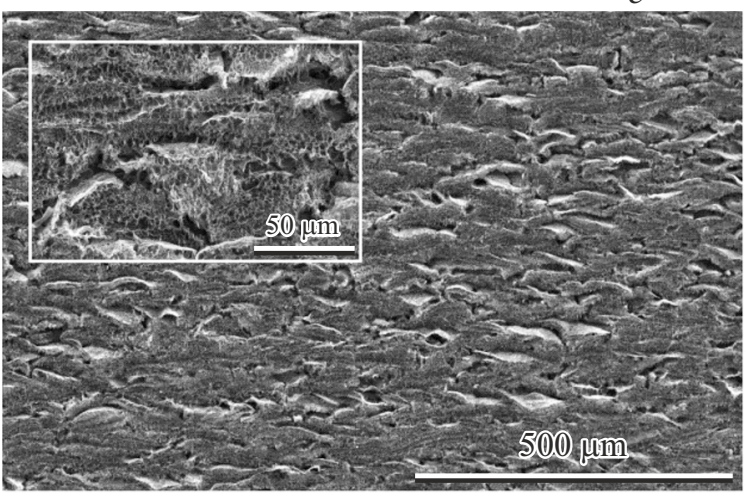

$b$

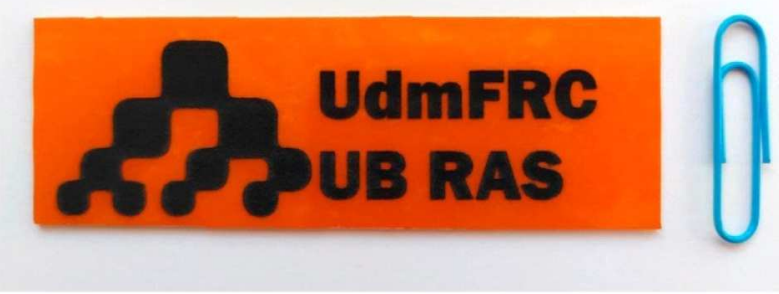

$d$

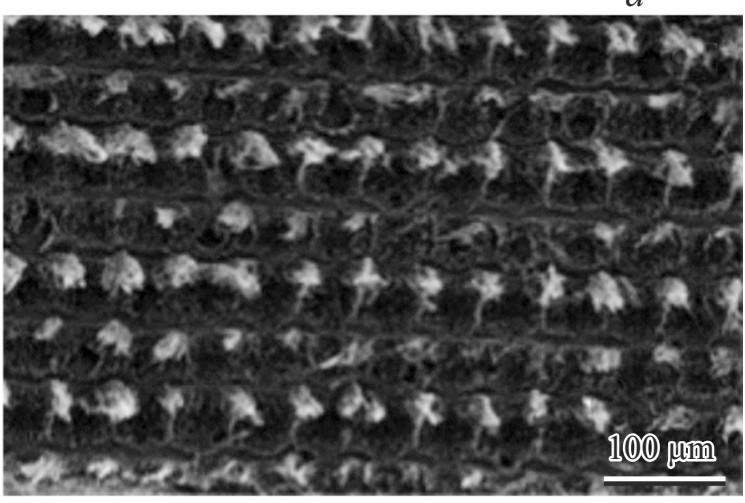

Pис. 1. $a-$ схема получения ЛИГ на ПИ-пленке с помощью построчного сканирования сфокусированного излучения СО ${ }_{2}$-лазера, $b$ - фотография ЛИГ на ПИ-пленке в форме эмблемы, $c$ - типичные изображения СЭМ поверхности ЛИГ, синтезированного с помощью непрерывного $\mathrm{CO}_{2}$ лазера. $d-$ изображение СЭМ поверхности ЛИГ, синтезированного с помощью импульснопериодического $\mathrm{CO}_{2}$-лазера (Reprinted from [28] with permission from Elsevier). Сканирование луча непрерывного $\mathrm{CO}_{2}$ лазера осуществлялось в плоскости $X Y$ при движении луча параллельно оси $Y$ слева направо, далее производилось смещение луча на шаг $25 \mu \mathrm{m}$ вдоль оси $+X$, далее движение луча лазера происходило в обратном направлении оси $Y$ (т. е. справа налево) и т.д.

казано, что сформированная таким образом пленочная структура ЛИГ обладает свойством генерации фототока по механизму эффекта увлечения [31-33] в широком спектральном диапазоне 266-1064 nm. Целью настоящей работы является более детальное исследование влияния режимов синтеза ЛИГ на поверхности ПИ-пленки с помощью построчного сканирования непрерывного излучения $\mathrm{CO}_{2}$-лазера на его структуру для получения ЛИГ с улучшенными свойствами, применимого, например, для создания микросуперконденсаторов.

\section{2. Объекты и методы исследования}

Для формирования образцов ЛИГ на поверхности ПИ-пленки (толщиной около $100 \mu \mathrm{m}$ ) использовалась лазерная установка, генерирующая непрерывное излучение на длине волны $10.6 \mu \mathrm{m}$ мощностью до $40 \mathrm{~W}$. Лазерный пучок фокусировался на поверхность ПИ-пленки линзой с фокусным расстоянием $51 \mathrm{~mm}$. Фокусирующая линза располагалась внутри конусообразного сопла и обдувалась воздушным потоком, направленным от линзы к перетяжке сфокусированного пучка. Это обеспечивало защиту поверхности линзы от загрязнения продуктами лазерного пиролиза ПИ-пленки. Диаметр сфокусированного лазерного пятна, измеренный по уровню $1 / \mathrm{e}^{2}$ интенсивности, на поверхности ПИ-пленки составлял
$190 \mu \mathrm{m}$. Мощность излучения, падающего на поверхность ПИ-пленки, варьировалась блоком питания лазерной установки. Оптимальная скорость сканирования, найденная в результате предварительных исследований, оставалась неизменной и составляла $220 \mathrm{~mm} / \mathrm{s}$. Схематичное изображение процесса лазерного пиролиза изображено на рис. $1, a$. Лазерное сопло сканировало, то есть построчно проходило поверхность ПИ, приклеенной к стеклянной подложке, вдоль оси $Y$, сдвигаясь после каждой строки на $25 \mu \mathrm{m}$ вдоль оси $X$. Благодаря автоматизированному управлению лазером, форма ЛИГ могла быть произвольной. Для примера на рис. $1, b$ показана фотография эмблемы Удмуртского федерального исследовательского центра УрО РАН (организации, с которой аффилированы авторы данной статьи), полученная за счет формирования ЛИГ на поверхности ПИ-пленки.

Морфология поверхности образцов характеризовалась с помощью оптического микроскопа Neophot 32, сопряженного с цифровой фотокамерой ToupCam U3ISPM18000KPA (USB3.0) [34], и сканирующего электронного микроскопа (СЭМ) (Thermo Fisher Scientific Quattro S). Анализ структуры ПИ-пленки и углеродных пленок, полученных в результате лазерного воздействия, проводился с помощью спектрометра комбинационного рассеяния света (KPC) (HORIBA HR800, длина волны возбуждения $632.8 \mathrm{~nm}$ ) при плотности мощности лазер- 
ного излучения $14 \mathrm{~kW} / \mathrm{cm}^{2}$. Все спектры КРС образцов ЛИГ, представленные в данной работе, приводятся после вычитания фона люминесценции. Помимо этого, спектры КРС синтезированных пленочных структур ЛИГ с разным количеством строк и количеством повторов сканирований нормированы на интенсивность D-полосы. Диагностика атомного состава и химических связей пленок, проводилась с помощью рентгенофотоэлектронного спектрометра (SPECS, $\mathrm{Mg} K_{\alpha}$ возбуждение при постоянной энергии пропускания энергоанализатора $15 \mathrm{eV}$ ). Сопротивление образцов измерялось по постоянному току при комнатной температуре.

\section{3. Результаты и обсуждение}

На рис. 1, $c$ представлены изображения поверхности пленки ЛИГ, синтезированной при мощности лазерного излучения $6.2 \mathrm{~W}$, полученные с помощью сканирующего электронного микроскопа. На рис. $1, c$ можно наблюдать строки, напоминающие бороздки на вспаханном поле, а на вставке к рис. 1,c отчетливо видна пористая структура, при этом размер пор достигает $1 \mu \mathrm{m}$.

Следует отметить, что структура поверхности ЛИГ, полученного с помощью непрерывного $\mathrm{CO}_{2}$-лазера, существенно отличается от структуры поверхности ЛИГ, полученного в [28] в результате воздействия импульснопериодического $\mathrm{CO}_{2}$-лазера (см. рис. $1, d$ ). Из сравнения фотографий, представленных на рис. $1, c, d$, видно, что синтез ЛИГ с помощью непрерывного лазерного излучения обеспечивает более однородную структуру вдоль линии сканирования лазерного луча, по сравнению с синтезом ЛИГ с помощью импульсно-периодического лазерного излучения. По данным рентгеновской фотоэлектронной спектроскопии (РФЭС) (см. рис. 2) в поверхностном слое типичной пленки ЛИГ присутствуют углерод (90.8 at.\%), кислород (7.5 at.\%) и азот (1.7 at.\%). Основной пик в C1s спектре относится к нефункционализированному углероду графена с энергией связи $284.4 \mathrm{eV}$. Высокоэнергетическая часть спектра связана с присутствием различных кислородсодержащих групп, в том числе эфирных, фенольных, карбонильных, карбоксильных и карбонатных групп, а также связей C-N. B O1s спектре наблюдаются составляющие от групп с двойной и одинарной связью между атомами углерода и кислорода, а также небольшая составляющая при $535.0 \mathrm{eV}$, которую можно отнести к адсорбированному на поверхности кислороду. В N1s-спектре наблюдаются составляющие от атомов азота пиридинового, пиррольного и замещающего (графитоподобного) типа, появившиеся в результате пиролиза ПИ, содержащей азот. Известно, что графен, содержащий такие азотные составляющие обладает более высоким потенциалом для создания микросуперконденсаторов за счет более высокой подвижности электронов, по сравнению с графеном не содержащим азот [35]. В целом, спектры РФЭС синтезированных пленочных структур ЛИГ хо-
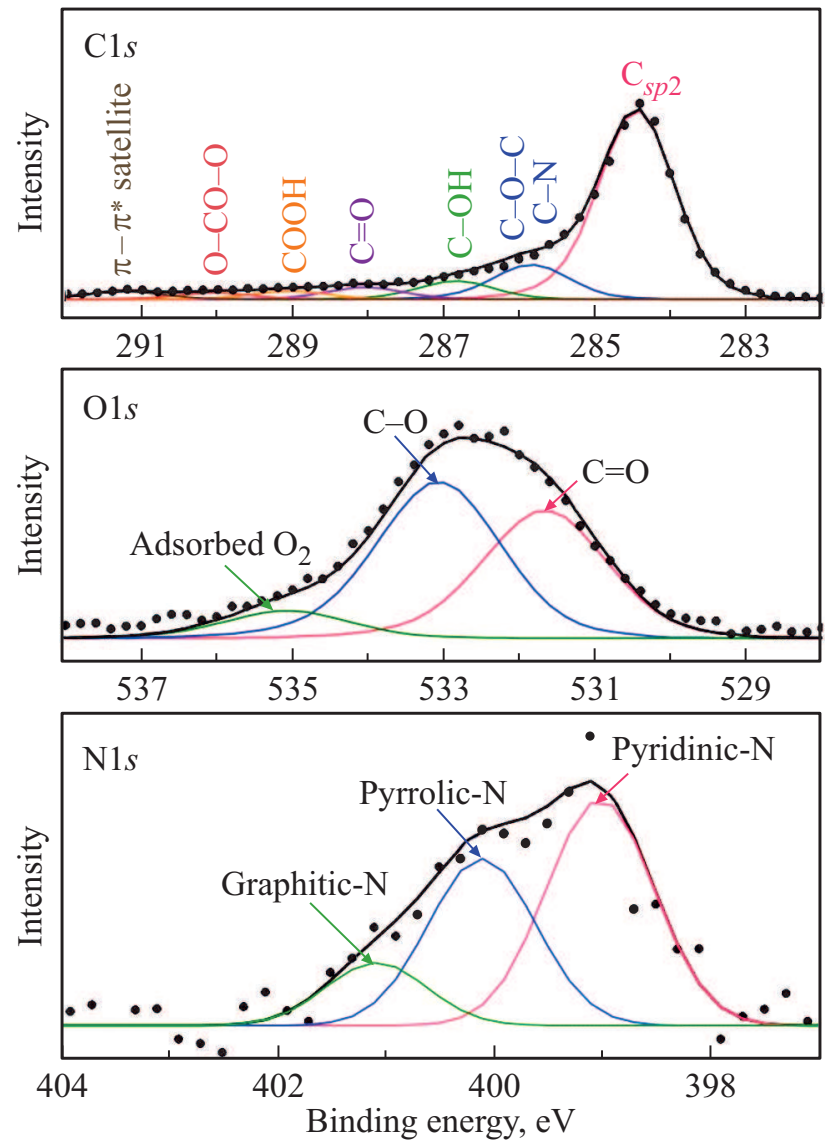

Рис. 2. C1s-, O1s- и N1s-спектры лазерно-индуцированного графена с указанием наиболее вероятных составляющих спектров, полученные с помощью рентенофотоэлектронного спектрометра. Доля неокисленного $s p^{2}$-углерода (с энергией связи $284.4 \mathrm{eV}$ ) составляет $68.5 \%$.

рошо согласуются с результатами исследований ЛИГ, полученными в других работах, например [36,37].

На рис. 3 представлены спектры КРС поверхности ПИ-пленки в зависимости от мощности излучения непрерывного $\mathrm{CO}_{2}$-лазера. На спектре КРС исходной, не облученной лазером ПИ-пленки, видны полосы, которые, исходя из литературных данных [38-41], можно отнести к дыхательной моде колебаний ароматического кольца $\left(1122 \mathrm{~cm}^{-1}\right)$, валентным колебаниям C-N $\left(1390 \mathrm{~cm}^{-1}\right), \mathrm{C}=\mathrm{C}\left(\sim 1600 \mathrm{~cm}^{-1}\right)$, и $\mathrm{C}=\mathrm{O}\left(1789 \mathrm{~cm}^{-1}\right)$. Известно, что при нагреве ПИ пленки до температуры $550^{\circ} \mathrm{C}$ происходит высвобождение кислорода и азота, при нагреве до $700^{\circ} \mathrm{C}$ происходит карбонизация, а при нагреве до $3000^{\circ} \mathrm{C}$ - графенизация $[28,42,43]$. При воздействии лазерным излучением мощностью $2 \mathrm{~W}$ (рис. 3, кривая 2) исчезают характерные для ПИ-полосы и появляются полосы D $\left(1350 \mathrm{~cm}^{-1}\right)$ и $\mathrm{G}\left(1590 \mathrm{~cm}^{-1}\right)$, что свидетельствует о начале процесса карбонизации. Полоса рассеяния D возникает из-за наличия дефектов в гексагональной структуре $s p^{2}$-атомов, и она отсутствует в спектрах КРС графита и графена, свободных от 


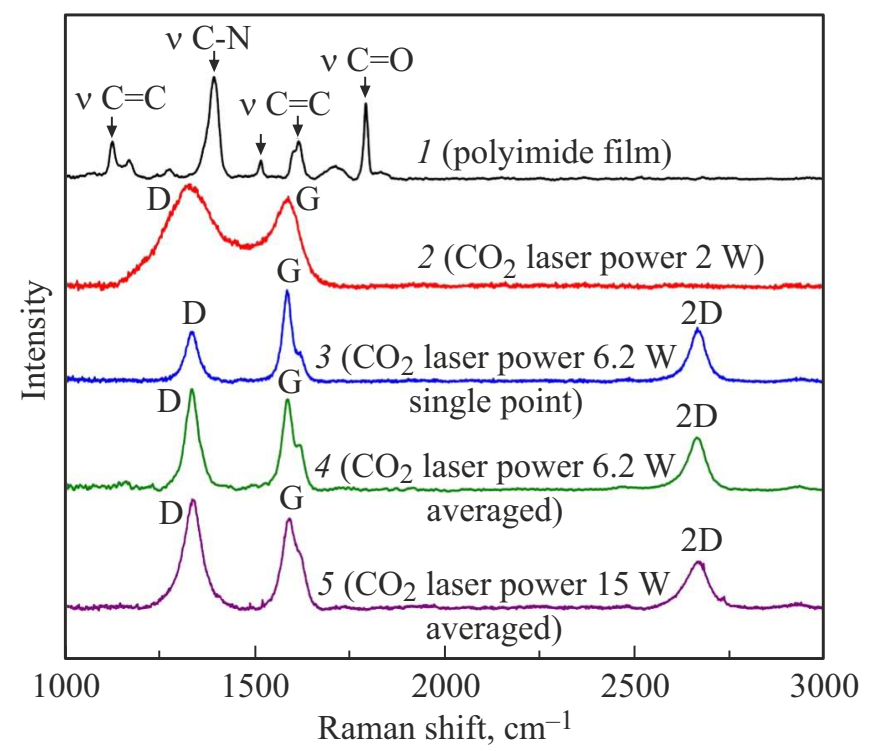

Рис. 3. Спектры КРС (1) ПИ-пленки и углеродной структуры в отдельно взятой точке, полученной на ПИ-пленке после воздействия лазерного излучения с мощностью (2) $2 \mathrm{~W}$, и (3) $6.2 \mathrm{~W}$, а также углеродной структуры, усредненный по 10 точкам, полученной на ПИ-пленке после воздействия лазерного излучения с мощностью (4) $6.2 \mathrm{~W}$ и (5) $15 \mathrm{~W}$.

дефектов [44]. G-полоса связана с продольной модой колебаний атомов углерода.

На рис. 3, кривая 3, показан спектр КРС участка ЛИГ, полученного при мощности $6.2 \mathrm{~W}$, снятый в произвольной точке на поверхности образца. Увеличение мощности лазерного воздействия, очевидно, повышает локальную температуру в области взаимодействия лазерного излучения с ПИ-пленкой, что приводит к появлению 2D-полосы $\left(\sim 2660 \mathrm{~cm}^{-1}\right)$. Эта полоса связана с потерей энергии кванта возбуждающего излучения на возбуждение поперечных оптических колебаний атомов углерода в графене [45]. Также наблюдаются сужение Dи G-полос и уменьшение отношения интенсивностей Dи $\mathrm{G}-п о л о с ~ I_{\mathrm{D} / \mathrm{G}}$. Такие изменения в спектре свидетельствуют об образовании графеновых слоев [28].

Отличительной особенностью спектра однослойного графена является интенсивная 2D-полоса, которая описывается одной кривой Лоренца с полушириной $25 \mathrm{~cm}^{-1}$, при этом $I_{2 \mathrm{D}} / I_{\mathrm{G}}>1$, где $I_{2 \mathrm{D}}$ и $I_{\mathrm{G}}-$ интенсивности полос 2D и $\mathrm{G}$ соответственно [44]. При увеличении количества слоев 2D-полоса преобразуется и представляет собой совокупность нескольких пиков, сдвинутых относительно друг друга по частотной шкале. В результате ширина результирующей 2D-полосы возрастает, а ее частотный сдвиг увеличивается. Одновременно существенно уменьшается ее интенсивность относительно G-полосы [46]. Из рис. 3, кривая 3 следует, что $2 \mathrm{D}$-полоса рассеяния имеет полуширину $\sim 45 \mathrm{~cm}^{-1}$, причем $I_{2 \mathrm{D}} / I_{\mathrm{G}} \approx 0.5$. Можно также отметить, что частотный сдвиг 2D-полосы в записанном спектре КРС совпадает с частотным сдвигом 2D-полосы графена, измеренным в работе [44] при лазерном возбуждении на длине волны $633 \mathrm{~nm}$. Таким образом, отношение $I_{2 \mathrm{D}} / I_{\mathrm{G}}$, частотный сдвиг и полуширина 2D-полосы свидетельствуют, что синтезированный нами углеродный материал состоит из множества графеновых слоев, расположенных случайным образом друг относительно друга $[45,46]$. Из рис. 3 , кривая 3 следует что $I_{\mathrm{D}} / I_{\mathrm{G}}$ составляет 0.5 . Это указывает на наличие дефектов в графеновых слоях [45].

Следует отметить, что усредненный спектр ЛИГ, синтезированного при мощности $6.2 \mathrm{~W}$ (рис. 3, кривая 4), отличается от единичного спектра, показанного на рис. 3, кривая 3. В усредненном спектре соотношение $I_{2 \mathrm{D}} / I_{\mathrm{G}} \approx 1$, и при этом наблюдается D-полоса, которая по положению, интенсивности и ширине на полувысоте идентична 2D-полосе на рис. 3, кривая 3. Таким образом, главное отличие этих спектров заключается в соотношении D- и G-полос, что можно объяснить неоднородностью структуры ЛИГ. При дальнейшем увеличении мощности лазера до $15 \mathrm{~W}$ увеличивается ширина полос D, G и $2 \mathrm{D}$, что свидетельствует о росте количества дефектов в структуре ЛИГ (см. рис. 3, кривая 5). Следует отметить, что при этой мощности наблюдается отслоение слоя ЛИГ от ПИ-подложки, и получение стабильной пленки становится затруднительным.

На всех спектрах ЛИГ присутствует $\mathrm{D}^{\prime}$-полоса $\left(\sim 1615 \mathrm{~cm}^{-1}\right)$, связанная с процессом двойного резонансного рассеяния [45]. Эта полоса может свидетельствовать о дефектах в графеновых слоях, связанных с азотом, наличие которого подтверждают данные РФЭС (см. рис. 2) [47]. Соотношение интенсивностей полос $\mathrm{D}$ и $\mathrm{D}^{\prime}$, которое указывает на тип дефектов, составляет $\sim 3.5$, что свидетельствует о дефектах преимущественно на краях графеновых слоев [48]. В целом, спектры, показанные на рис. 3, (кривые 2, 3 и 4) являются характерными для ЛИГ $[14,15,17,49,50]$.

Сканирование поверхности пучком лазера диаметром около $190 \mu \mathrm{m} \mathrm{c}$ шагом $25 \mu \mathrm{m}$ означает, что лазерный луч будет многократно проходить одну и ту же область пленки, что очевидным образом должно влиять на морфологию и структуру получаемого материала. Для исследования этого вопроса на поверхности ПИ были синтезированы образцы ЛИГ, в которых количество строк, сформированных в результате сканирования лазера при мощности лазерного излучения $6.2 \mathrm{~W}$, составляло от одной до пяти.

На рис. 4 показаны изображения поверхности этих образцов, полученные с помощью оптического микроскопа, и изображения СЭМ поперечного среза этих же образцов. Можно заметить, что ширина полосы увеличивается с увеличением количества строк (она составляет $\sim 200 \mu \mathrm{m}$ для одной строки, и $\sim 300 \mu \mathrm{m}$ для пяти строк). Тем не менее, для одной строки характерно образование структур, напоминающих хлопья (см. рис. 4,a). В разрезе такие хлопья хорошо видны на рис. 4,f. После образования второй строки хлопьев становится заметно меньше, что хорошо видно 

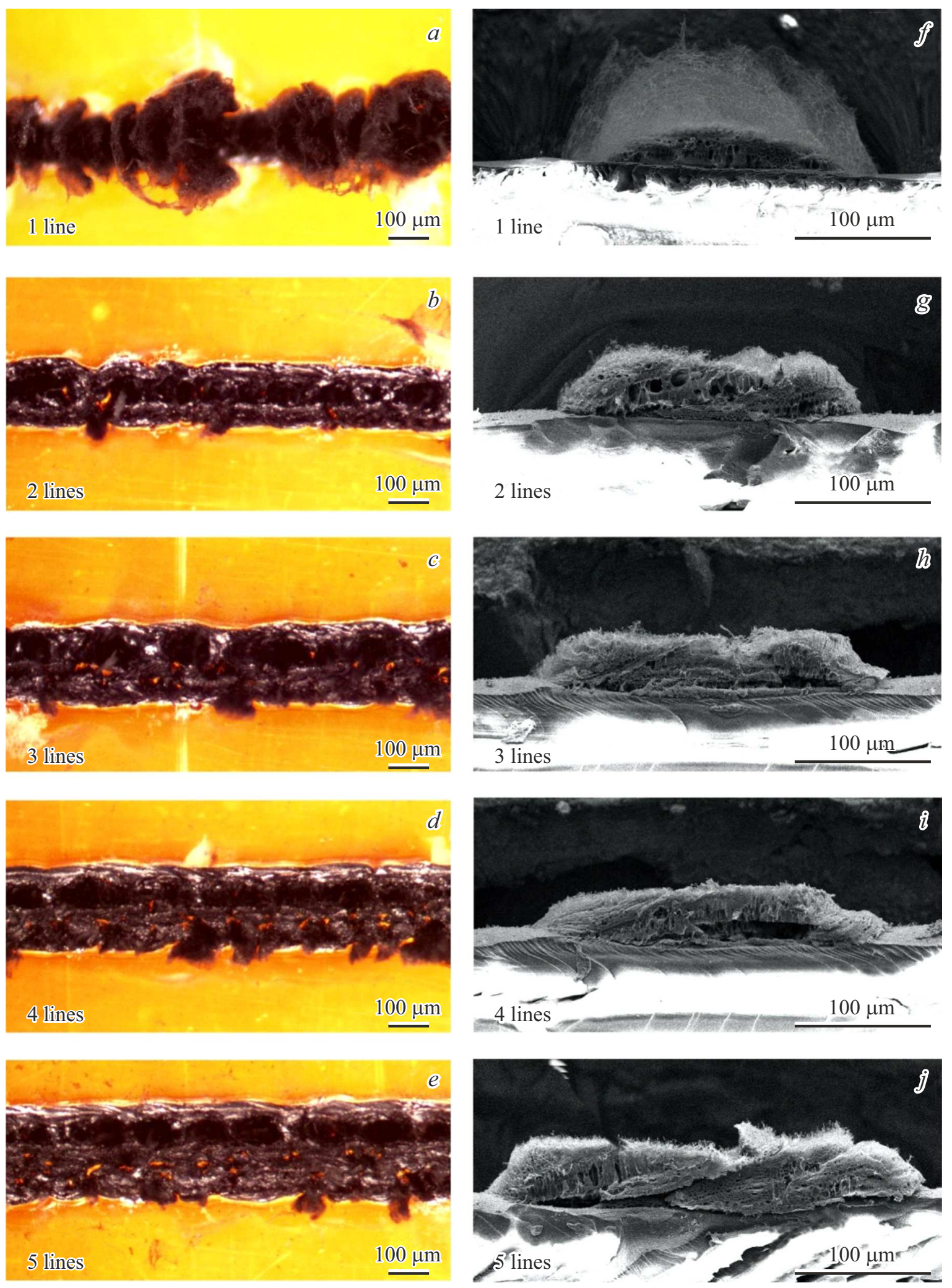

Рис. 4. $a-e-$ оптические изображения поверхности полос ЛИГ, сформированных на поверхности ПИ-пленки построчным сканированием лазерным лучом с разным количеством строк и $f-j$ - соответствующие СЭМ-изображения торцевого среза полос ЛИГ 

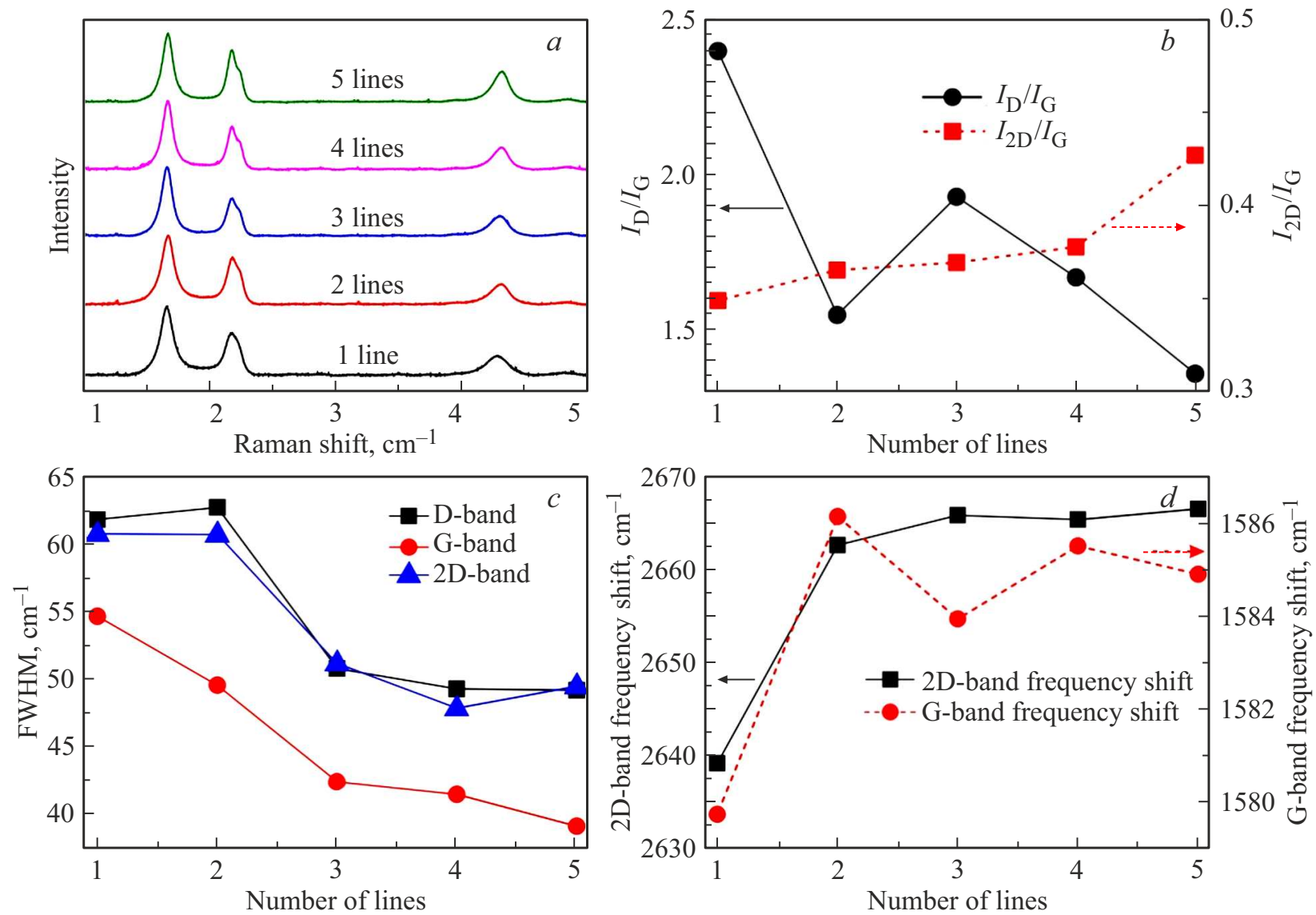

Рис. 5. $a-$ спектры КРС протяженных полос ЛИГ, сформированных построчным сканированием лазерным лучом с количеством строк от 1 до 5, усредненные по 20 спектрам, измеренных поперек полос с шагом $10 \mu \mathrm{m}$. Спектры нормированы на интенсивность D-полосы. Зависимости $(b)$ отношения интенсивности D- и G-полос (круги), 2D- и G-полос (квадраты), (c) ширины на полувысоте (FWHM) D-, G-, 2D-полос и $(d)$ положения G- (круги) и 2D-полос (квадраты) от количества строк в полосе ЛИГ.

на рис. $4, b, g$. Поскольку каждая следующая строка проходит через предыдущую большей своей площадью, наиболее вероятно, что это может объясняться тем, что эти хлопья сдуваются воздушным потоком (исходящим из лазерного сопла), защищающим фокусирующую линзу от загрязнений. Для исследования структурных различий полос ЛИГ с разным количеством строк были измерены их спектры КРС. Измерения проводились поперек полосы с шагом $10 \mu \mathrm{m}$, начиная от края и заканчивая через $200 \mu \mathrm{m}$ (ширина полосы с одной строкой) от точки начала. Такое расстояние выбрано для того, чтобы при усреднении учитывалось одинаковое количество точек для каждой полосы.

На рис. 5, $a$ представлены спектры КРС (усредненные по 20 измеренным спектрам) полос ЛИГ, сформированных построчным сканированием лазерным лучом с разным количеством строк. Из рис. 5, $а$ видно, что наблюдается уменьшение количества дефектов в структуре ЛИГ с увеличением количества строк. Это проявляется в уменьшении отношения интенсивностей D- и G-полос (см. рис. $5, b)$. Так, для образца с одной строкой $I_{\mathrm{D}} / I_{\mathrm{G}}$ составляет 2.39, тогда как для образца с пятью строками этот параметр равен 1.35. Отношение интенсив- ностей $2 \mathrm{D}$-полосы к G-полосе $I_{2 \mathrm{D}} / I_{\mathrm{G}}$, наоборот, растет с 0.35 до 0.42 для одной и пяти строк, соответственно. Одновременно происходит уменьшение полуширин D-, G-, 2D-полос (рис. 5,c), а также смещение 2D-полосы в красную область с увеличением количества строк (рис. $5, d$ ). Все это указывает на уменьшение количества дефектов в графеновых слоях и рост степени графенизации.

Такое влияние числа строк на структуру ЛИГ можно объяснить следующим образом. При лазерном облучении ПИ-пленки одной строки вдоль оси $Y$ (см. рис. 1, $a$ ) происходит частичная графенизация поверхности, и формируется полоса, шириной около $200 \mu \mathrm{m}$ (см. рис. 4, $а$ ). Дальше лазер смещается вдоль оси $X$ на $25 \mu \mathrm{m}$ и движется вдоль оси $Y$ в обратную сторону. При этом большая часть площади пучка повторно проходит через частично графенизированный слой, тем самым, увеличивая степень графенизации. Следующая строка снова большей своей площадью проходит через две предыдущих строки и еще больше увеличивает степень графенизации. Таким образом, по площади первой строки лазер проходит около 8 раз, при этом наименьшее количество пересечений образуется на краях полос. По этой причине, а так же по 

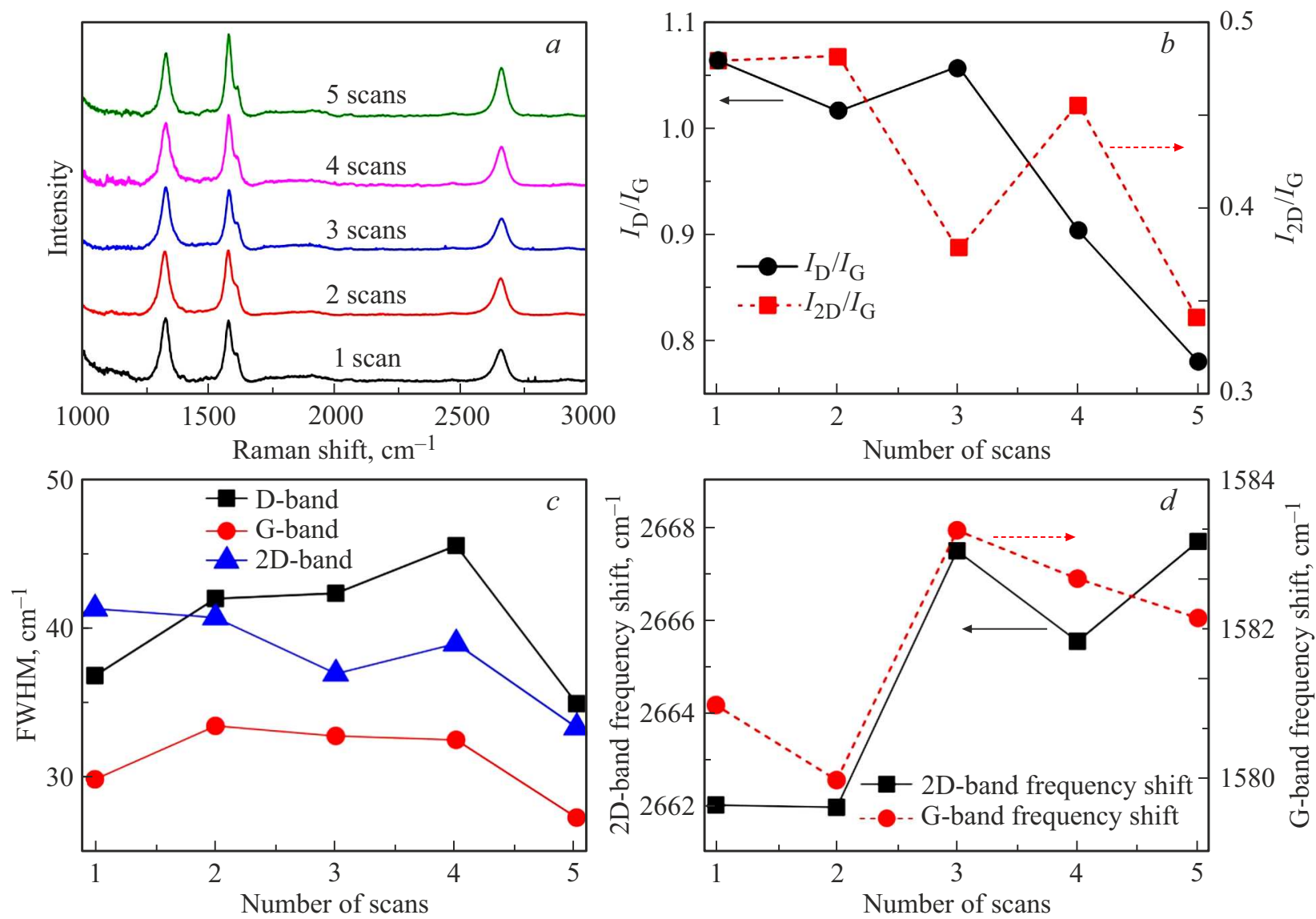

Рис. 6. $a-$ спектры КРС полос ЛИГ, сформированных построчных сканированием лазерным лучом с разным количеством повторов лазерного сканирования, усредненные по 20 спектрам. Спектры нормированы на интенсивность D-полосы. Зависимости (b) отношения интенсивности D- и G-полос (круги), 2D- и G-полос (квадраты), (c) ширины на полувысоте (FWHM) D-, G-, 2D-полос и $(d)$ положения G- (круги) и 2D-полос (квадраты) от количества повторов лазерного сканирования.

причине того, что интенсивность лазерного пучка минимальна на его краях, мы наблюдали, что по периметру отдельной строки часто встречаются спектры, в которых отсутствует 2D-полоса, и присутствуют широкие D- и G-полосы, т. е. спектры аморфного углерода, схожие со спектром на рис. 3, кривая 2.

Учитывая полученные знания о том, что повторное прохождение лазером по уже сформированной строке ЛИГ увеличивает степень графенизации, можно предположить, что повторное сканирование лазером целого образца ЛИГ позволит получить ЛИГ с улучшенными свойствами. Для этого были приготовлены 5 образцов ЛИГ на ПИ-пленке, отличающиеся количеством повторов сканирований. На первом этапе все образцы построчно сканировались в обычном режиме, в результате на каждом образце был сформирован слой ЛИГ. На втором этапе выполнялось еще одно построчное сканирование лазером по уже сформированному слою ЛИГ на четырех оставшихся образцах. На третьем этапе сканировались три из предыдущих четырех образцов, а на четвертом - два, на пятом - один. Таким образом, были сформированы пять образцов ЛИГ с разным количеством повторов сканирований: от одного до пяти.
На рис. 6, $а$ представлены спектры КРС образцов ЛИГ, сформированных построчных сканированием лазерного луча с разным количеством повторов сканирований, усредненные по 20 спектрам.

Видно, что с увеличением количества повторов отношения $I_{\mathrm{D}} / I_{\mathrm{G}}$ и $I_{2 \mathrm{D}} / I_{\mathrm{G}}$ уменьшаются (см. рис. $6, b$ ). Так, для образца ЛИГ, полученного однократным сканированием, $I_{\mathrm{D}} / I_{\mathrm{G}}=1.06$ и $I_{2 \mathrm{D}} / I_{\mathrm{G}}=0.48$, в то время как для образца ЛИГ, полученного с пятью повторами сканирования, $I_{\mathrm{D}} / I_{\mathrm{G}}=0.78$ и $I_{2 \mathrm{D}} / I_{\mathrm{G}}=0.34$. Полуширины D-, G-, 2D-полос остаются примерно на одном и том же уровне (см. рис. 6,c), а частотные сдвиги G- и 2D-полос незначительно смещаются в красную область (см. рис. 6,d).

Интересно отметить, что в дополнительных экспериментах при увеличении расстояния между строками, степень графенизации поверхности ПИ-пленки уменьшается, на что указывает увеличение $I_{\mathrm{D}} / I_{\mathrm{G}}$ и уменьшение $I_{2 \mathrm{D}} / I_{\mathrm{G}}$. При этом, какое бы ни было расстояние между строками, при повторном лазерном сканировании степень графенизации растет неизменно.

Одним из ключевых параметров пленочных структур ЛИГ (с точки зрения разработки и создания на их основе 


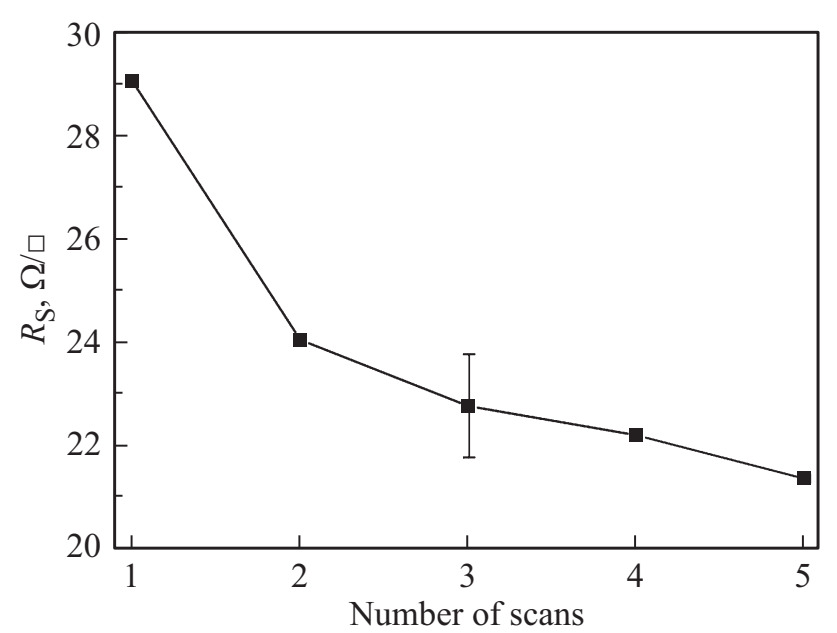

Рис. 7. Зависимость поверхностного сопротивления пленочной структуры ЛИГ от количества повторов сканирований.

различных электротехнических устройств и датчиков) является удельное сопротивление на квадрат (англ. „sheet resistance“, далее для простоты - поверхностное сопротивление $R_{\mathrm{S}}$ ). В связи с этим, было исследовано влияние многократного лазерного сканирования на $R-\mathrm{S}$. Для этого были синтезированы 25 образцов с пленочной структурой ЛИГ в форме прямоугольника размерами $0.5 \times 3.0 \mathrm{~cm}$. При этом первые пять образцов ЛИГ были получены в результате однократного сканирования, следующая группа из 5 образцов - в результате двукратного сканирования, третья группа из 5 образцов - в результате трехкратного сканирования и т.д.

На рис. 7 приведена зависимость $R_{\mathrm{S}}$ от количества повторов сканирования, где каждая точка была получена после усреднения измеренного поверхностного сопротивления пяти образцов, синтезированных при одинаковых условиях. Из нее видно, что $R_{\mathrm{S}}$ пленочной структуры ЛИГ, синтезированной в результате однократного сканирования лазерного луча по поверхности ПИ, составляет 29.0 $\Omega / \square$. При последующих повторах сканирования $R_{\mathrm{S}}$ монотонно уменьшается. После пятикратного сканирования $R_{\mathrm{S}}$ уменьшается до $21.3 \Omega / \square$, т. е. более чем на $25 \%$ по сравнению со значением, полученным при однократном сканировании. Зависимость поверхностного сопротивления ЛИГ от количества повторов сканирования в целом коррелирует с соответствующей зависимостью $I_{\mathrm{D}} / I_{\mathrm{G}}$, показанной на рис. $6, a$, т.е. поверхностное сопротивление ЛИГ падает вместе с количеством дефектов в его структуре. Исследования с помощью оптического микроскопа и СЭМ показывают, что при увеличении количества повторов сканирования наблюдается увеличение толщины слоя ЛИГ (до 40\%), что является одной из причин синхронного уменьшения $R_{\mathrm{S}}$. Очевидно, что все это должно влиять на электрохимические свойства синтезируемых пленочных структур ЛИГ, исследование которых представляет несомненный интерес, но выходит за рамки данной работы.

\section{4. Заключение}

Проведенные исследования показывают, что пиролиз ПИ-пленки при построчном сканировании сфокусированного пучка непрерывного $\mathrm{CO}_{2}$-лазера (диаметр перетяжки $190 \mu \mathrm{m})$ при мощности $2 \mathrm{~W}$ приводит к превращению ПИ-пленки в аморфную углеродную структуру. При повышении мощности до $6.2 \mathrm{~W}$ начинает происходить графенизация поверхности, т.е. образование ЛИГ. При более высокой мощности $(>6.2 \mathrm{~W})$ также происходит образование ЛИГ, однако, при этом повышается риск отслоения ЛИГ от ПИ-пленки. Спектроскопия КРС показывает, что количество дефектов в отдельной строке ЛИГ существенно больше, чем в полосе ЛИГ, представляющую собой суперпозицию из строк ЛИГ, частично накладывающихся друг на друга $\left(I_{\mathrm{D}} / I_{\mathrm{G}}\right.$ уменьшается с 2.39 до 1.35). Многократное лазерное сканирование по одному и тому же участку поверхности ПИ-пленки с определенной площадью также понижает количество дефектов в слое ЛИГ $\left(I_{\mathrm{D}} / I_{\mathrm{G}}\right.$ уменьшается с 1.06 для слоя ЛИГ, полученного однократным сканированием, до 0.78 для слоя ЛИГ, полученного пятикратным сканированием). Показано, что увеличение количества повторов лазерной обработки до пяти сопровождается уменьшением поверхностного сопротивления синтезируемой пленочной структуры ЛИГ более чем на $25 \%$. Полученные результаты могут быть использованы для дальнейшего усовершенствования технологии синтеза электропроводящих углеродных пленочных структур для изготовления различных датчиков и электронных устройств.

\section{Финансирование работы}

Работа выполнена в рамках государственного задания Министерства образования и науки Российской Федерации (государственный регистрационный номер 1021032422167-7-1.3.2) и при финансовой поддержке Российского научного фонда (проект 19-72-00071). Исследование выполнено с использованием оборудования центра совместного использования „Центра физических и физико-химических методов анализа и изучения свойств и поверхностных характеристик наноструктур, материалов и изделий“ УдмФИЦ УрО РАН.

\section{Конфликт интересов}

Авторы заявляют об отсутствии конфликта интересов.

\section{Список литературы}

[1] Y. Yang, Y. Song, X. Bo, J. Min, O.S. Pak, L. Zhu, M. Wang, J. Tu, A. Kogan, H. Zhang, T.K. Hsiai, Z. Li, W. Gao. Nat. Biotechnol. 38, 217 (2020).

[2] B. Wang, A. Facchetti. Adv. Mater. 31, 1 (2019).

[3] N. Kurra, Q. Jiang, P. Nayak, H.N. Alshareef. Nano Today 24, 81 (2019). 
[4] C. Wang, C. Wang, Z. Huang, S. Xu. Adv. Mater. 30, 1 (2018).

[5] C. Zhang, Z. Peng, C. Huang, B. Zhang, C. Xing, H. Chen, H. Cheng, J. Wang, S. Tang. Nano Energy 81, 105609 (2021).

[6] V. Strauss, K. Marsh, M.D. Kowal, M. El-Kady, R.B. Kaner. Adv. Mater. 30, 1 (2018).

[7] J. Ye, H. Tan, S. Wu, K. Ni, F. Pan, J. Liu, Z. Tao, Y. Qu, H. Ji, P. Simon, Y. Zhu. Adv. Mater. 30, 1801384 (2018).

[8] Y. Zhang, L. Zhang, K. Cui, S. Ge, X. Cheng, M. Yan, J. Yu, H. Liu. Adv. Mater. 30, 1 (2018).

[9] Z. Wei, D. Wang, S. Kim, S.-Y. Kim, Y. Hu, M.K. Yakes, A.R. Laracuente, Z. Dai, S.R. Marder, C. Berger, W.P. King, W.A. de Heer, P.E. Sheehan, E. Riedo. Science 328, 1373 (2010).

[10] S. Stankovich, D.A. Dikin, R.D. Piner, K.A. Kohlhaas, A. Kleinhammes, Y. Jia, Y. Wu, S.T. Nguyen, R.S. Ruoff. Carbon N. Y. 45, 1558 (2007).

[11] D.A. Sokolov, K.R. Shepperd, T.M. Orlando. J. Phys. Chem. Lett. 1, 2633 (2010).

[12] R. You, Y. Liu, Y. Hao, D. Han, Y. Zhang, Z. You. Adv. Mater. 32, 1901981 (2020).

[13] Z. Wan, E.W. Streed, M. Lobino, S. Wang, R.T. Sang, I.S. Cole, D.V. Thiel, Q. Li. Adv. Mater. Technol. 3, 1 (2018).

[14] R. Ye, Y. Chyan, J. Zhang, Y. Li, X. Han, C. Kittrell, J.M. Tour. Adv. Mater. 29, 1702211 (2017).

[15] R. Ye, D.K. James, J.M. Tour. Adv. Mater. 31, 1803621 (2019).

[16] D. Zhai, Y. Yang, Z. Geng, B. Cui, R. Zhao. IEEE Trans. Terahertz Sci. Technol. 8, 719 (2018).

[17] J. Lin, Z. Peng, Y. Liu, F. Ruiz-Zepeda, R. Ye, E.L.G.G. Samuel, M.J. Yacaman, B.I. Yakobson, J.M. Tour. Nature Commun. 5, 5714 (2014).

[18] Y. Ding, Z. Cheng, X. Zhu, K. Yvind, J. Dong, M. Galili, H. Hu, N.A. Mortensen, S. Xiao, L.K. Oxenløwe. Nanophotonics 9, 317 (2020).

[19] P. Zaccagnini, D. di Giovanni, M.G. Gomez, S. Passerini, A. Varzi, A. Lamberti. Electrochim. Acta 357, 136838 (2020).

[20] Y. Wang, G. Wang, M. He, F. Liu, M. Han, T. Tang, S. Luo. Small 2103322, 1 (2021).

[21] L. Huang, L. Ling, J. Su, Y. Song, Z. Wang, B.Z. Tang, P. Westerhoff, R. Ye. ACS Appl. Mater. Interfaces 12, 51864 (2020).

[22] L. Cheng, W. Guo, X. Cao, Y. Dou, L. Huang, Y. Song, J. Su, Z. Zeng, R. Ye. Mater. Chem. Front. (2021).

[23] H. Wang, Z. Zhao, P. Liu, X. Guo. Biosensors 12, 55 (2022).

[24] S. Kaur, S. Karmakar, K.M. Devi, R.K. Varshney, D.R. Chowdhury. Optik 248, 168073 (2021).

[25] M.K. Smith, D.X. Luong, T.L. Bougher, K. Kalaitzidou, J.M. Tour, B.A. Cola. Appl. Phys. Lett. 109, 253107 (2016).

[26] X. Yu, N. Li, S. Zhang, C. Liu, L. Chen, S. Han, Y. Song, M. Han, Z. Wang. J. Power Sources 478, 229075 (2020).

[27] M.G. Stanford, C. Zhang, J.D. Fowlkes, A. Hoffman, I.N. Ivanov, P.D. Rack, J.M. Tour, A. Ho, I.N. Ivanov, P.D. Rack, J.M. Tour. ACS Appl. Mater. Interfaces 12, 10902 (2020).

[28] L.X. Duy, Z. Peng, Y. Li, J. Zhang, Y. Ji, J.M. Tour. Carbon N. Y. 126, 472 (2018).

[29] К.Г. Михеев, Р.Г. Зонов, Д.Л. Булатов, А.Е. Фатеев, Г.М. Михеев. Письма в ЖТФ 46, 51 (2020).

[30] K.G. Mikheev, R.G. Zonov, T.N. Mogileva, A.E. Fateev, G.M. Mikheev. Opt. Laser Technol. 141, 107143 (2021).

[31] E.L. Ivchenko. Optical Spectroscopy of Semiconductor Nanostructures. Springer (2004).
[32] G.M. Mikheev, A.S. Saushin, V.V. Vanyukov, K.G. Mikheev, Y.P. Svirko. Nanoscale Res. Lett. 12, 39 (2017).

[33] K.G. Mikheev, A.S. Saushin, R.G. Zonov, A.G. Nasibulin, G.M. Mikheev. J. Nanophotonics 10, 012505 (2015).

[34] Г.М. Михеев, Р.Г. Зонов, А.Ю. Попов, Д.Г. Калюжный. Приборы и техника эксперимента 46, 3, 164 (2003).

[35] E. Haque, M.M. Islam, E. Pourazadi, M. Hassan, S.N. Faisal, A.K. Roy, K. Konstantinov, A.T. Harris, A.I. Minett, V.G. Gomes. RSC Adv. 5, 30679 (2015).

[36] E.R. Mamleyev, S. Heissler, A. Nefedov, P.G. Weidler, N. Nordin, V.V. Kudryashov, K. Länge, N. MacKinnon, S. Sharma. Npj Flex. Electron. 3, 2 (2019).

[37] Z. Wan, M. Umer, M. Lobino, D. Thiel, N.T. Nguyen, A. Trinchi, M.J.A. Shiddiky, Y. Gao, Q. Li. Carbon N.Y. 163, 385 (2020).

[38] X.J. Gu. Appl. Phys. Lett. 62, 1568 (1993).

[39] D.W. Mayo, F.A. Miller, R.W. Hannah. Course Notes on the Interpretation of Infrared and Raman Spectra. John Wiley \& Sons, Inc., Hoboken, NJ, USA (2004).

[40] S. Devasahayam, D.J.T. Hill, J.W. Connell. J. Appl. Polym. Sci. 101, 1575 (2006).

[41] G. Socrates. Infrared and Raman Characteristic Group Frequencies: Tables and Charts. 3rd ed. Wiley (2004).

[42] A. Lamberti, M. Serrapede, G. Ferraro, M. Fontana, F. Perrucci, S. Bianco, A. Chiolerio, S. Bocchini. 2D Mater. 4, 035012 (2017).

[43] P. Zaccagnini, C. Ballin, M. Fontana, M. Parmeggiani, S. Bianco, S. Stassi, A. Pedico, S. Ferrero, A. Lamberti. Adv. Mater. Interfaces 8, 2101046 (2021).

[44] A.C. Ferrari. Solid State Commun. 143, 47 (2007).

[45] L.M. Malard, M.A. Pimenta, G. Dresselhaus, M.S. Dresselhaus. Phys. Rep. 473, 51 (2009).

[46] A.C. Ferrari, J.C. Meyer, V. Scardaci, C. Casiraghi, M. Lazzeri, F. Mauri, S. Piscanec, D. Jiang, K.S. Novoselov, S. Roth, A.K. Geim. Phys. Rev. Lett. 97, 1 (2006).

[47] Z. Zafar, Z.H. Ni, X. Wu, Z.X. Shi, H.Y. Nan, J. Bai, L.T. Sun. Carbon N. Y. 61, 57 (2013).

[48] A. Eckmann, A. Felten, A. Mishchenko, L. Britnell, R. Krupke, K.S. Novoselov, C. Casiraghi. Nano Lett. 12, 3925 (2012).

[49] X. Wang, Y. Cui, Y. Tao, H. Yang, J. Zhao. J. Electron. Mater. 10, (2020).

[50] R. Ye, D.K. James, J.M. Tour. Acc. Chem. Res. 51, 1609 (2018).

Редактор Т.Н. Василевская 